\title{
Phenotypic and Cellular
} Characteristics of a Stromal Vascular Fraction/Extracellular Matrix Gel Prepared Using Mechanical Shear Force on Human Fat

OPEN ACCESS

Edited by:

Arnaud Scherberich,

University Hospital of Basel,

Switzerland

Reviewed by:

Jeffrey Gimble,

Obatala Sciences, United States

Joris Van Dongen,

University Medical Center Groningen,

Netherlands

*Correspondence:

Jindou Jiang

jiangjindou1@163.com

Specialty section:

This article was submitted to

Tissue Engineering and Regenerative

Medicine,

a section of the journal

Frontiers in Bioengineering and

Biotechnology

Received: 06 December 2020

Accepted: 03 February 2021

Published: 26 February 2021

Citation:

Ye Y, Zou J, Tan M, Hu K and

Jiang J (2021) Phenotypic

and Cellular Characteristics of a

Stromal Vascular

Fraction/Extracellular Matrix Gel

Prepared Using Mechanical Shear

Force on Human Fat.

Front. Bioeng. Biotechnol. 9:638415.

doi: 10.3389/fbioe.2021.638415

\author{
Yuan Ye, Jingjiang Zou, Meijun Tan, Kuikui Hu and Jindou Jiang* \\ Department of Plastic and Cosmetic Surgery, Guangdong Women and Children Hospital, Guangzhou, China
}

The retention of fat-derived grafts remains a challenge for regenerative medicine. Fat aspirates from patients undergoing liposuction were prepared into standard Coleman fat grafts or further isolated using mechanical shear force to prepare a stromal vascular fraction (SVF)/extracellular matrix (ECM) gel. The retention rate of the SVF/ECM gel was significantly higher than that of the Coleman fat at 3, 14, 28, and 60 days following transplantation on the backs of nude mice. The viscosity of the fat was directly proportional to the shearing force. Although the mechanical isolation did not affect the total number of cells, it significantly decreased the number of living cells. Flow cytometry showed a greater number of mesenchymal stem cells, supra-adventitial (SA)-adipose stromal cells (ASCs), and adipose-derived stem cells but a lower number of endothelial progenitor cells in the SVF/ECM gel than in the Coleman fat. Thus, mechanical isolation of fat can increase the pluripotency of adipocytes, which can improve graft retention in cell therapy.

Keywords: SVF/ECM gel, Coleman fat, shear force, tissue regeneration, stem cells, flow cytometry

\section{INTRODUCTION}

Fat grafting is currently considered the standard procedure for repairing soft-tissue defects, which has been widely used in the fields of plastic, reconstructive, and esthetic surgery (Cohen et al., 2017; Rohrich et al., 2018; Cansancao et al., 2019; Li X. et al., 2019). However, the instability of long-term effects after fat transplantation limits the clinical application of fat grafting (Qiu et al., 2016). The success of fat transplantation is affected by several factors such as donor age, donor body mass index, donor site, and the methods of fat treatment and transplantation (Cucchiani and Corrales, 2016; Tuin et al., 2016). Currently, the fat treatment method is considered to be the most important factor influencing transplantation success (Canizares et al., 2017; Streit et al., 2017). Accordingly, the optimization of fat transplantation has attracted increasing attention in the field of regenerative medicine. Novel fat transplantation schemes have been developed over the last decade to minimize intervention, with the goal of destroying several mature adipocytes while retaining the regeneration and differentiation ability of stem cells (Yao et al., 2017; van Dongen et al., 2018; Tonnard et al., 2020).

Tonnard et al. (2013) used an emulsification technique to chylose fat, resulting in "nanofat," providing a new mode of fat treatment. Nanofat is rich in growth factors and regeneration-related cells with the potential to play a role in cell therapy but is not suitable for filling the soft-tissue volume (Tonnard et al., 2013, 2020; Uyulmaz et al., 2018; Chen et al., 2019; Verpaele et al., 2019; 
Pallua and Kim, 2020). Yao et al. (2017) also used mechanical shear force to obtain a gelatinous fat that was named stromal vascular fraction (SVF)/extracellular matrix (ECM) gel, which also contained a rich source of regeneration-related cells and ECM, demonstrating the potential for cell therapy (Yao et al., 2017). Both the emulsification and shear force methods involve complete physical approaches without requiring an enzyme.

Accumulating evidence indicates that activating cells through mechanical signals in the extracellular microenvironment can mediate a series of signaling pathways through cytoskeleton rearrangement, thereby altering the proliferation and differentiation abilities of progenitor cells (Nava et al., 2012; Sun et al., 2012; Li Y. et al., 2019; Chen et al., 2020; Zhang et al., 2020). An SVF/ECM gel is a type of Coleman fat obtained using standard Coleman technology following centrifugation and is further mechanically isolated. The mechanically disrupted lipoaspirate product is acquired through minimal manipulation methods, resulting in a tissue SVF (tSVF) (Trivisonno et al., 2019). We hypothesized that mechanical isolation not only results in a simple physical effect of thinning the fat but can also ultimately improve fat retention by affecting the regeneration ability of cells in the SVF/ECM gel, i.e., the application of different shear forces may cause biological changes in related cells in the grafts to ultimately affect the regeneration success. The SVF/ECM gel contains abundant vascular matrix fragments, which have been widely used in cell therapy. However, little is known regarding the specific proportion of and changes in regeneration-related cell subsets in an SVF/ECM gel prepared using mechanical shear force.

In this study, we compared the expression of cell surface antigens and the proportion of cell subsets under different shear forces (Coleman fat without shear force and SVF/ECM gel with mechanical isolation via application of shear force) and evaluated the retention rate of fat grafts under these conditions in a mouse model. Our study showed that efficient and stable fat retention may be possible through SVF/ECM gel transplantation without any additional cells, thus achieving ideal capacity filling. Additionally, we found that shear force is positively correlated with viscosity, and the viscosities of Coleman fat and SVM/ECM gel differ. These findings can provide a theoretical basis for the clinical application of autologous fat.

\section{MATERIALS AND METHODS}

\section{Fat Sample Acquisition and Processing}

To reduce the influence of potential interference factors, we obtained fat aspirates from the abdomen of seven non-obese women undergoing liposuction without metabolic diseases. The mean ( \pm standard deviation) age of the patients was $34.0 \pm 3.6$ years with a body mass index of $20.1 \pm 1.6 \mathrm{~kg} / \mathrm{m}^{2}$. This study received approval from the institutional ethics committee of Guangdong Woman and Children Hospital (no. 201801005). All patients provided informed consent for the use of their samples for research purposes. The samples were divided into two groups: Coleman fat group and SVF/ECM gel group. Coleman fat was prepared following the classic Coleman technology method
(Coleman, 2006). In brief, a 10-mL syringe was used to connect the tonsil porous liposuction needle with a diameter of $3 \mathrm{~mm}$ and a diameter of $1 \mathrm{~mm}$ for negative pressure suction, and the obtained liposuction aspirate was further centrifuged at 1,200 $\times g$ for $3 \mathrm{~min}$. The upper oil and lower water layers were removed, and the fat tissue in the middle was retained without applying shear force; it was collected as the Coleman fat and set as the blank control group. The preparation method of the SVF/ECM gel followed the method described by Yao et al. (2017). In brief, a $10-\mathrm{mL}$ syringe was used to connect the liposuction needle with a diameter of $3 \mathrm{~mm}$ and a sharp aperture of $1 \mathrm{~mm}$ for negative pressure suction. Liposuction was performed at a suction pressure of -0.75 atm. The obtained liposuction aspirate was further centrifuged at $1,200 \times g$ for $3 \mathrm{~min}$ to remove the moisture in the lower layer while carefully adding $0.5 \mathrm{~mL}$ of oil. Two $10-\mathrm{mL}$ syringes were used with a female-to-female Luer lock connector with an inner diameter of $2.4 \mathrm{~mm}$ to push against each other for $1 \mathrm{~min}$ at a mutual pushing speed of $10 \mathrm{~mL} / \mathrm{s}$. The enmeshed fat was obtained and centrifuged again at 2,000 $\times g$ for $3 \mathrm{~min}$. After removing water and oil, the middle fat tissue was retained and was collected as the SVF/ECM gel. Figure 1 shows a schematic of the specific operation steps for each group.

\section{Animal Model Establishment and Experimental Grouping}

This study received approval from the Institutional Animal Care and Use Committee of Guangzhou Medical University according to the rules of the Committee on Animal Research and Ethics (no. GY2018-018). Six- to eight-week-old male specific pathogenfree BALB/C nude mice purchased from Guangdong Medical Experimental Animal Center were selected as the transplant recipients. The breeding and operation of nude mice were conducted in the experimental animal center of Guangzhou Medical University. For the experimental group, human adipose tissue was divided into two groups: Coleman fat group and SVF/ECM gel group. Each fat type was prepared according to the descriptions outlined in Section "Fat Sample Acquisition and Processing" (Figure 1). The nude mice were anesthetized with an intraperitoneal injection of pentobarbital sodium $(50 \mathrm{mg} / \mathrm{kg})$. The procedure was performed in 25 nude mice per group, and Coleman fat $(0.1 \mathrm{~mL})$ and SVF/ECM gel $(0.1 \mathrm{~mL})$ were both transplanted onto the backs of each nude mouse simultaneously to establish a fat transplantation nude mouse model using a 1$\mathrm{mL}$ syringe with a blunt-tipped, 14-gauge infiltration cannula. Therefore, the total number of samples was 50. All nude mice were euthanized, and the grafted fat was harvested after $0,3,14$, 28 , and 60 days ( $n=5$ at each time point) (Figure 2). Grafted samples were fixed in $4 \%$ paraformaldehyde for $24 \mathrm{~h}$, then dehydrated, and paraffin-embedded for histological examination.

\section{General and Histological Observation of Fat Grafts}

The general and histological changes in fat samples were observed and recorded at days $3,14,28$, and 60 after fat transplantation, and the long-term retention rate was evaluated. Five-micrometer-thick specimens were prepared and stained 

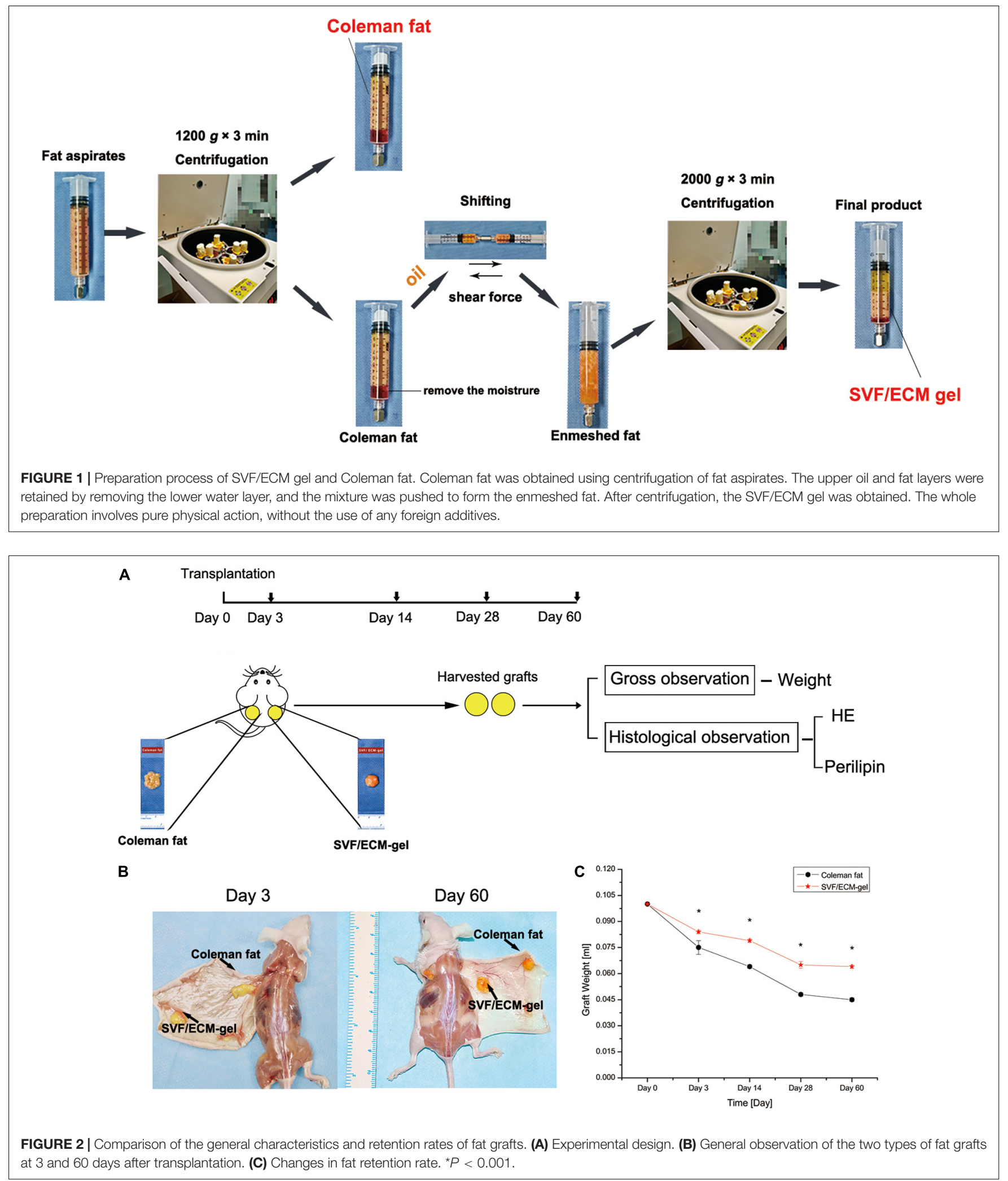

with hematoxylin and eosin (HE). Images were captured with an Olympus BX51 microscope and Olympus Dp71 digital camera for analysis. The following antibodies were used for immunostaining: guinea pig anti-mouse perilipin as the primary antibody (diluted 1:400; Progen, Heidelberg, Germany) and Alexa fluor 647-labeled goat anti-guinea pig immunoglobulin 
G (diluted 1:2,000; Abcam) as the secondary antibody. The cell nucleus was labeled with DAPI (diluted 1:200; Sigma, St. Louis, MO, United States). All data were collected and analyzed by two observers.

\section{Calculation of Fluid Shear Force}

Since the SVF/ECM gel was obtained through mechanical isolation of Coleman fat, the shear stresses of Coleman fat and the SVF/ECM gel were compared by measuring their respective viscosities to evaluate the effect of different degrees of shear stress on the cell behavior of the grafts. The viscosity of the fluid was measured using a DV-IPRIME Viscometer (Brookfield Engineering Laboratories, Inc., Boulevard, MA, United States). First, the Reynolds coefficient ( $\mathrm{Re}$ ) was calculated to evaluate whether the fluid flow characteristics were laminar or turbulent, using the following formula: $\operatorname{Re}=2 \mathrm{q} / \pi \nu \mathrm{R}$, where $\mathrm{q}$ represents the volume flow of the measured medium, $v$ represents the kinematic viscosity of the medium, and $\mathrm{R}$ represents the radius of the adapter pipe connecting the syringe. A Re value $<2,300$ represents laminar flow, Re of 2,300-4,000 represents a transition state, and $\operatorname{Re}>4,000$ indicates turbulent flow. If laminar flow was detected, the shear stress $(\tau)$ of the fat sample was further calculated with the formula $\tau=4 \mu \mathrm{Q} / \pi \mathrm{R} 3$, where $\mu$ represents dynamic viscosity, calculated as $\nu \times \rho$ in which $\rho$ is the approximate water density (Banyard et al., 2016).

\section{Separation of the SVF}

Coleman fat and the SVF/ECM gel were prepared from 10$\mathrm{mL}$ fat samples as described above and further processed for obtaining the SVF as described previously (Banyard et al., 2016; Yao et al., 2017). In brief, the samples were washed with phosphate-buffered saline repeatedly to remove the blood. Thereafter, 0.075\% collagenase I (Sigma-Aldrich Co., St. Louis, MO, United States) was added to the fat tissue samples and digested in a constant temperature shaker at $37^{\circ} \mathrm{C}$ for $30 \mathrm{~min}$. The sample was then mixed repeatedly two to three times; neutralized in a complete medium of equal volume high-glucose Dulbecco's modified Eagle medium, 10\% fetal bovine serum, and $1 \%$ penicillin-streptomycin (Sigma); and centrifuged at $800 \times g$ for $5 \mathrm{~min}$. The cell pellets were then resuspended and filtered through a $100-\mu \mathrm{m}$ mesh. Red blood cell lysate (Sigma) was added to minimize erythrocyte contamination and incubated at $37^{\circ} \mathrm{C}$ for $5 \mathrm{~min}$, followed by a final centrifugation at $800 \times g$ for $5 \mathrm{~min}$. The aqueous portions were removed after each centrifugation step. The pellets were then resuspended in control media and subjected to staining and analysis.

\section{Analysis of the Number, Density, and Activity of SVF Cells}

Stromal vascular fraction cells in the fresh suspension from the two groups of samples were isolated and counted using fluorescence double staining. Additionally, for apoptosis analysis, the cells were incubated with fluorescein isothiocyanateconjugated Annexin V (Caltag, Burlingame, CA, United States) at $37^{\circ} \mathrm{C}$ for $20 \mathrm{~min}$ and analyzed directly with propidium iodide (PI) using flow cytometry.

\section{Phenotype Analysis of SVF Cells}

Fluorescence-activated cell sorting was used to analyze the cell surface markers in the SVF cell suspensions using the following monoclonal antibodies: mouse anti-human CD45-PECytm5, CD31-PE-Cytm7, CD34-FITC, CD146-PE, CD13-PE, and CD73-FITC (BD Biosciences). The single-cell suspension was placed in a polystyrene tube on ice. An LSR II (BD Biosciences) flow cytometer was used to detect the cell density and cell surface markers in the SVF suspension.

\section{Proportion of Cell Subsets in the SVF Cell Suspension}

Multiple fluorescence staining was performed in the fresh SVF cell suspensions obtained from Coleman fat and the SVF/ECM gel. The number and proportion of cell subsets in SVF cell suspensions were analyzed through detection of their markers: endothelial progenitor cells (EPCs), $\mathrm{CD} 45^{-} \mathrm{CD} 31^{+} \mathrm{CD} 34^{+} \mathrm{CD} 146^{+}$; adipose-derived stem cells (ASCs), $\quad \mathrm{CD}_{4}{ }^{-} \mathrm{CD} 31^{-} \mathrm{CD} 13^{+} \mathrm{CD}^{+} 3^{+}$; transitional cells, $\mathrm{CD} 45^{-} \mathrm{CD} 31^{-} \mathrm{CD} 34^{+} \mathrm{CD} 146^{+}$; pericytes, $\mathrm{CD} 45^{-} \mathrm{CD} 31^{-} \mathrm{CD} 34^{-}$ $\mathrm{CD}_{146^{+}}$; and supra-adventitial (SA)-adipose stromal cells (ASCs), $\mathrm{CD}^{-} 5^{-} \mathrm{CD} 31^{-} \mathrm{CD} 34^{+} \mathrm{CD}^{-} 6^{-}$(Zimmerlin et al., 2013; Guo et al., 2016).

\section{Statistical Analysis}

Data are expressed as mean \pm standard deviation and were analyzed using SPSS 20.0. Independent sample $t$-tests were used to compare the data of the two groups at a single time point. $P<0.05$ was considered as a statistically significant difference.

\section{RESULTS}

\section{General Observations and Long-Term Retention of Grafts}

At 3, 14, 28, and 60 days after fat transplantation, the retention rate of the SVF/ECM gel graft was significantly higher than that of the Coleman fat graft at all time points (all $P<0.001$; Figure 2).

\section{Histological Observation of Grafts}

Hematoxylin and eosin staining showed that 60 days after transplantation, the SVF/ECM gel formed a mature lobular fat structure, whereas some oil drops remained in the central area of the Coleman fat graft sites (Figure 3A). The adipocytes were labeled using perilipin, which is a protein that coats lipid droplets only in viable adipocytes (Eto et al., 2012; Kato et al., 2014; Doi et al., 2015). Three days after transplantation, compared with those in the Coleman fat grafts, the adipocytes in the SVF/ECM gel grafts were necrotic and the negative area of perilipin significantly increased due to destruction by the applied shear force. Sixty days after transplantation, a large number of new adipocytes with positive perilipin staining were found at the SVF/ECM gel transplant site, and the size and morphology were regular, whereas several negative areas of perilipin were observed at the Coleman fat graft site (Figure 3B). 

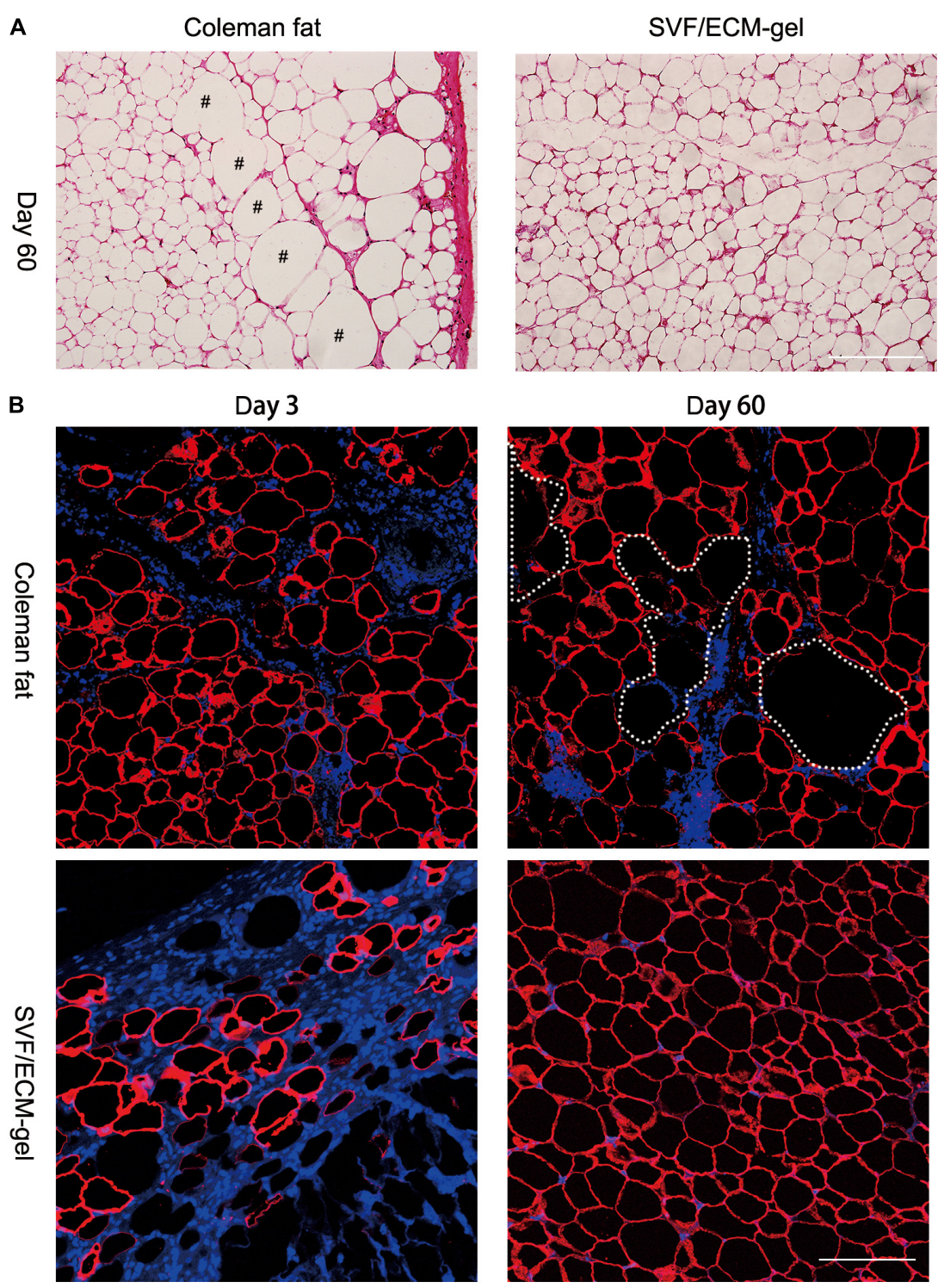

Perilipin DAPI

FIGURE 3 | Histological changes in the transplanted fat. (A) Hematoxylin and eosin staining on day 60 after transplantation showing that the SVF/ECM gel formed a mature lobular fat structure (right), whereas some oil droplets remained in the Coleman fat grafts (left); scale bars $=200 \mu \mathrm{m}$. (B) Fluorescence staining showing that 3 days after transplantation, the adipocytes in the SVF/ECM gel grafts were necrotic, and the negative area of perilipin was significantly increased (lower left), compared to the Coleman fat grafts (upper left); 60 days after transplantation, a large number of new adipocytes with positive perilipin staining were found in the SVF/ECM gel grafts (lower right), with many negative areas (upper right) of perilipin in the Coleman fat grafts; scale bars $=100 \mu \mathrm{m}$.

\section{Relationship Between Viscosity and Shear Force}

The relationship between shear force and kinematic viscosity in the process of mechanical isolation from standard Coleman fat to the SVF/ECM gel was analyzed. The fat was in an enmeshed state before SVF/ECM gel centrifugation; thus, the kinematic viscosity of Coleman fat was much higher than that of the SVF/ECF gel measured under the same connector radius and volume flow of $1.2 \mathrm{~mm}$ and $10 \mathrm{~mL} / \mathrm{s}$, respectively. The Re values were 65.9 and 18.9 for the SVF/ECF gel and Coleman fat, respectively, indicating laminar flow. The shear force of Coleman fat was also much higher than that of the SVF/ECM gel (Figure 4). These results demonstrated that with the decrease in viscosity, the ability to produce shear force was reduced.

\section{Number, Density, and Activity of SVF Cells}

As shown in Figure 5, there was no significant difference in the number of SVF cells obtained from the Coleman fat and SVF/ECM gel $(P=0.681)$, and the final volume of the SVF/ECM 


\section{Shear Force as Function of Viscosity}

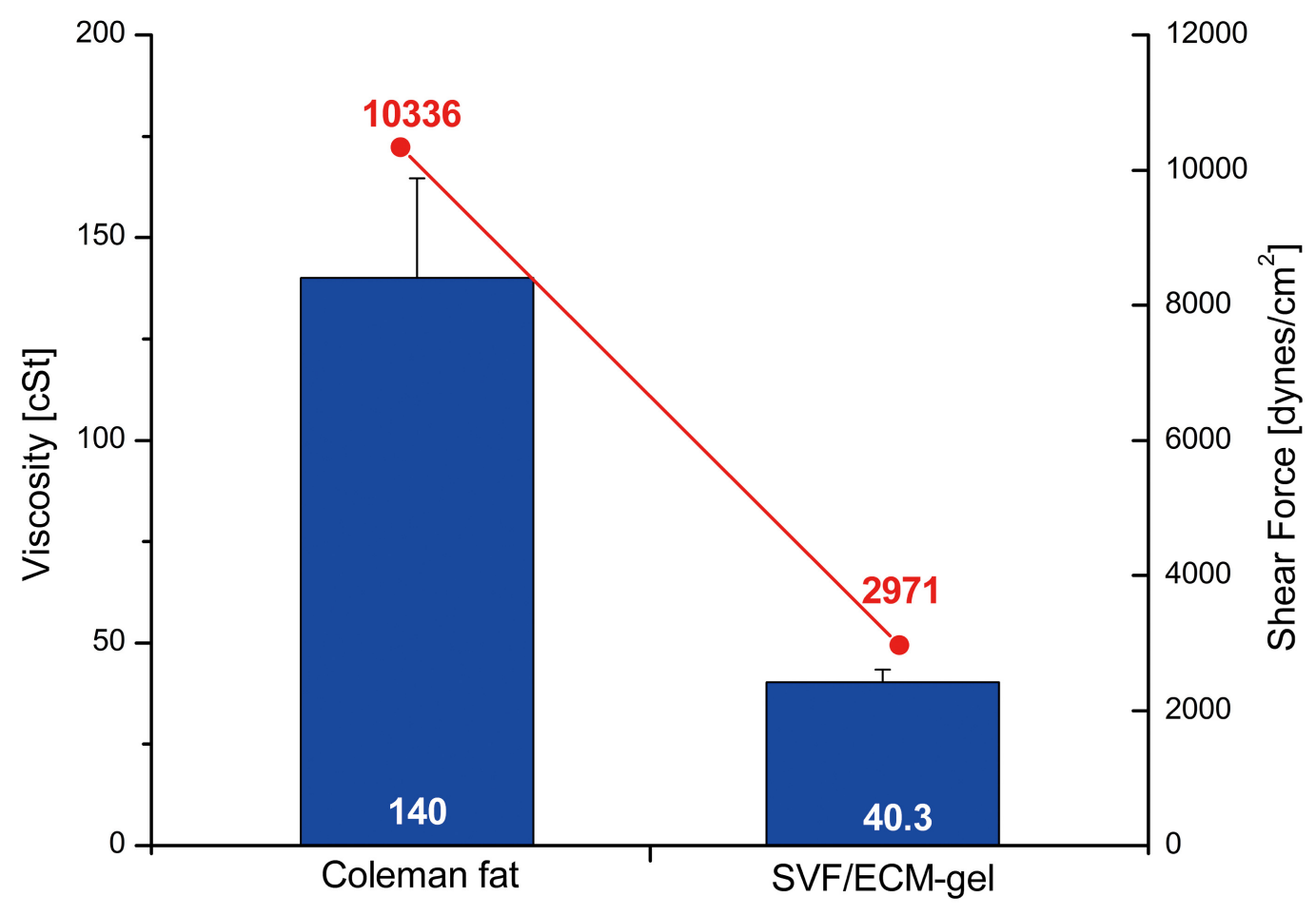

Viscosity [cSt] $-O-$ Shear Force [dynes $/ \mathrm{cm}^{2}$ ]

FIGURE 4 | Relationship between shear force (line) and kinematic viscosity (columns) of different fat types. The SVF/ECM gel was obtained by mechanical isolation of the initial Coleman fat through the application of shear force.

gel was about one-quarter that of the Coleman fat $(P<0.05)$. In addition, the cell density of the SVF/ECM gel graft increased significantly compared to that of the Coleman fat graft $(P<0.05)$. However, the number of living cells in the Coleman fat graft site was significantly higher than that in the SVF/ECM gel graft site $(P<0.05$; Figure 6).

\section{Phenotype Analysis of SVF Cells in Grafts}

Compared with the Coleman fat grafts, the SVF/ECM gel grafts showed a more than threefold increase in the levels of the general stem cell marker CD34 $(P<0.05)$, mesenchymal stem cell markers, and hematopoietic markers $(P<0.05)$ (Figure 7$)$. Thus, the expression of these markers in the SVF/ECM gel grafts was induced by shear stress, becoming significantly higher than that in the Coleman fat grafts without shear stress.

\section{Proportion of Cell Subsets in the SVF Cell Suspension}

The proportions of ASCs $\left(\mathrm{CD} 45^{-} \mathrm{CD} 31^{-} \mathrm{CD} 13^{+} \mathrm{CD}^{+}\right)$ and SA-ASCs $\left(\mathrm{CD}_{4} 5^{-} \mathrm{CD} 31^{-} \mathrm{CD} 34^{+} \mathrm{CD} 146^{-}\right)$increased significantly in the SVF/ECM grafts compared to those in the Coleman fat grafts. However, the proportions of EPCs $\left(\mathrm{CD}_{4} 5^{-} \mathrm{CD} 31^{+} \mathrm{CD} 34^{+} \mathrm{CD} 146^{+}\right)$and peripheral cells
$\left(\mathrm{CD} 45^{-} \mathrm{CD} 31^{-} \mathrm{CD} 34^{-} \mathrm{CD} 146^{+}\right)$decreased significantly in the SVF/ECM grafts compared to those in the Coleman fat grafts. There was no significant difference in the proportion of transitional cells $\left(\mathrm{CD} 45^{-} \mathrm{CD} 31^{-} \mathrm{CD} 34^{+} \mathrm{CD} 146^{+}\right)$between the two graft types (Figure 8).

\section{DISCUSSION}

Development of new strategies to improve the retention rate following fat transplantation has become a research hotspot. The activity of adipocytes is affected by fat preparation and processing methods (Oranges et al., 2019; Sese et al., 2019; Wei et al., 2019). The key factor contributing to the fat retention rate is the extent of retainment of the integrity and activity of the adipocyte structure in the fat graft (Yuan et al., 2015). In recent years, Kato et al. (2014) proposed the three-zone theory of fat transplantation, based on two older theories: the cell survival theory, which states that only one to three layers of mature adipocytes could survive in grafts due to the lack of early blood supply and oxygen content, and the host cell replacement theory, indicating that new adipocytes are formed almost exclusively by the differentiation of ASCs (Billings and May, 1989). Several studies subsequently confirmed 


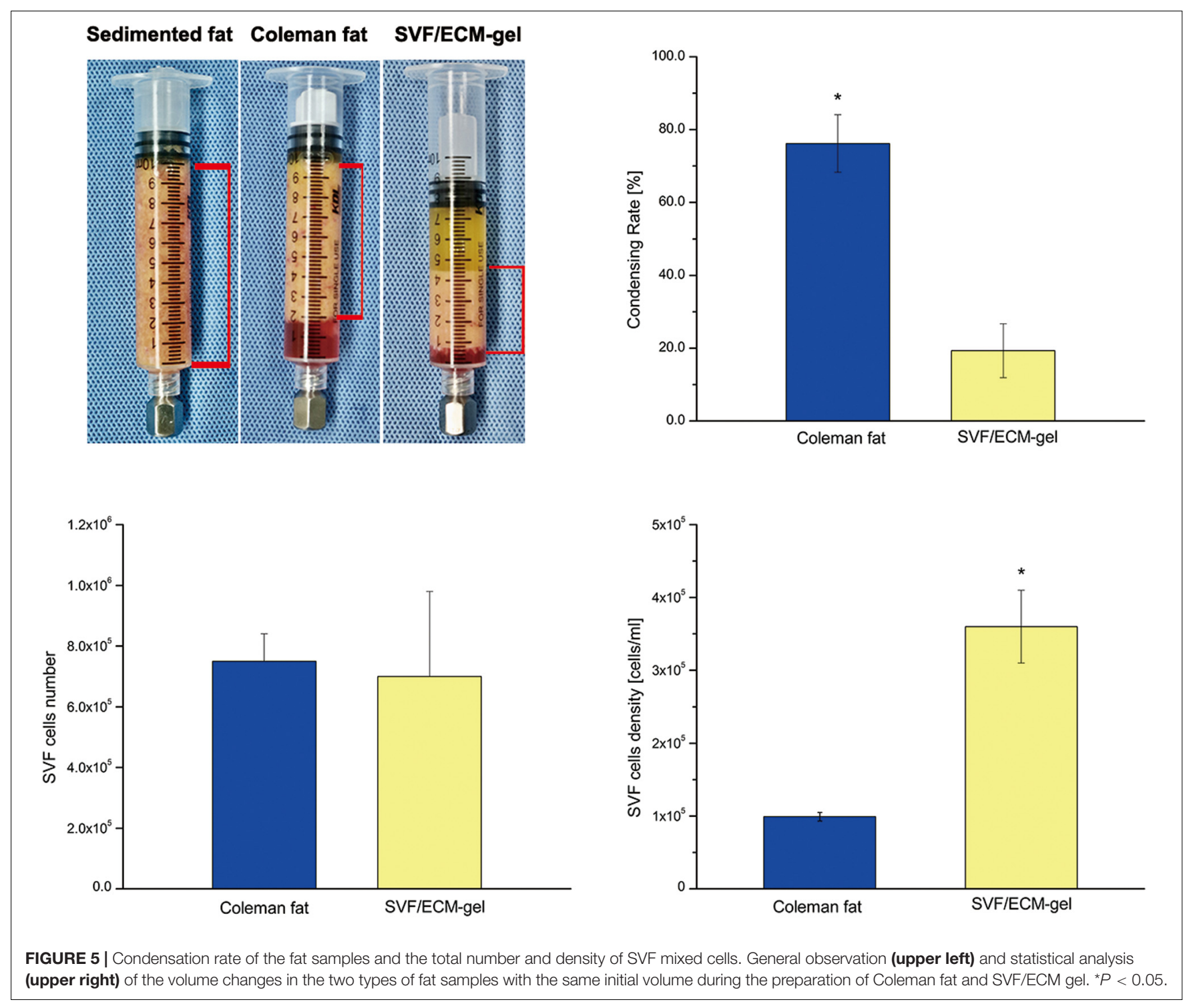

this theory of fat regeneration (Doi et al., 2015; Dong et al., 2015; Ye et al., 2017; Tang et al., 2018). Therefore, the key to fat regeneration is to increase the content of SVF cells and ASCs. At present, the cell-assisted fat transplantation technology Lipotransfer involves the addition of regenerated cells (ASCs or SVF cells), which has proven to significantly improve the retention rate of fat grafts in small-scale fat transplantation (Zhang et al., 2016; Zhou et al., 2016; Gontijode-Amorim et al., 2017; Li et al., 2017; Cai et al., 2018; Bashir et al., 2019; Hong et al., 2019; Kim et al., 2019). However, this technology is limited based on safety challenges such as enzyme digestion, contamination, and possible promotion of tumor recurrence in the process of regeneration related to the steps of cell separation; these have hindered its clinical use (Fraser et al., 2011; Krumboeck et al., 2013; Orbay et al., 2018; Gebremeskel et al., 2019).

In this study, we improved the technology of SVF/ECM gel preparation introduced by Yao et al. (2017), in which mechanical shear force was used to prepare the SVF/ECM gel, which is also commonly described as tSVF (Trivisonno et al., 2019). With no filtering technique, more ECM is saved, leading to an increase in the relative numbers of regenerative cells such as ASCs, pericytes, and SA-ASCs that attached around vessels embedded in the ECM compared to the results obtained in the original study on this technique (Yao et al., 2017). Moreover, compared to Coleman fat, there was no significant difference in the number of mixed cells isolated from the SVF/ECM. Interestingly, we found a higher number of living cells in the Coleman fat grafts than in the SVF/ECM gel. Since the total number of cells was the same in the SVF/ECM gel and Coleman fat, given that the same initial volume of fat samples was used, and the lowest layer of blood was discarded after centrifugation in both cases, the mechanical isolation step involved in SVF/ECM gel processing likely did not affect the live cell number. However, since the volume of mature adipocytes is much larger than that of other cells, we suspect that mechanical isolation mainly destroys the cell membrane 


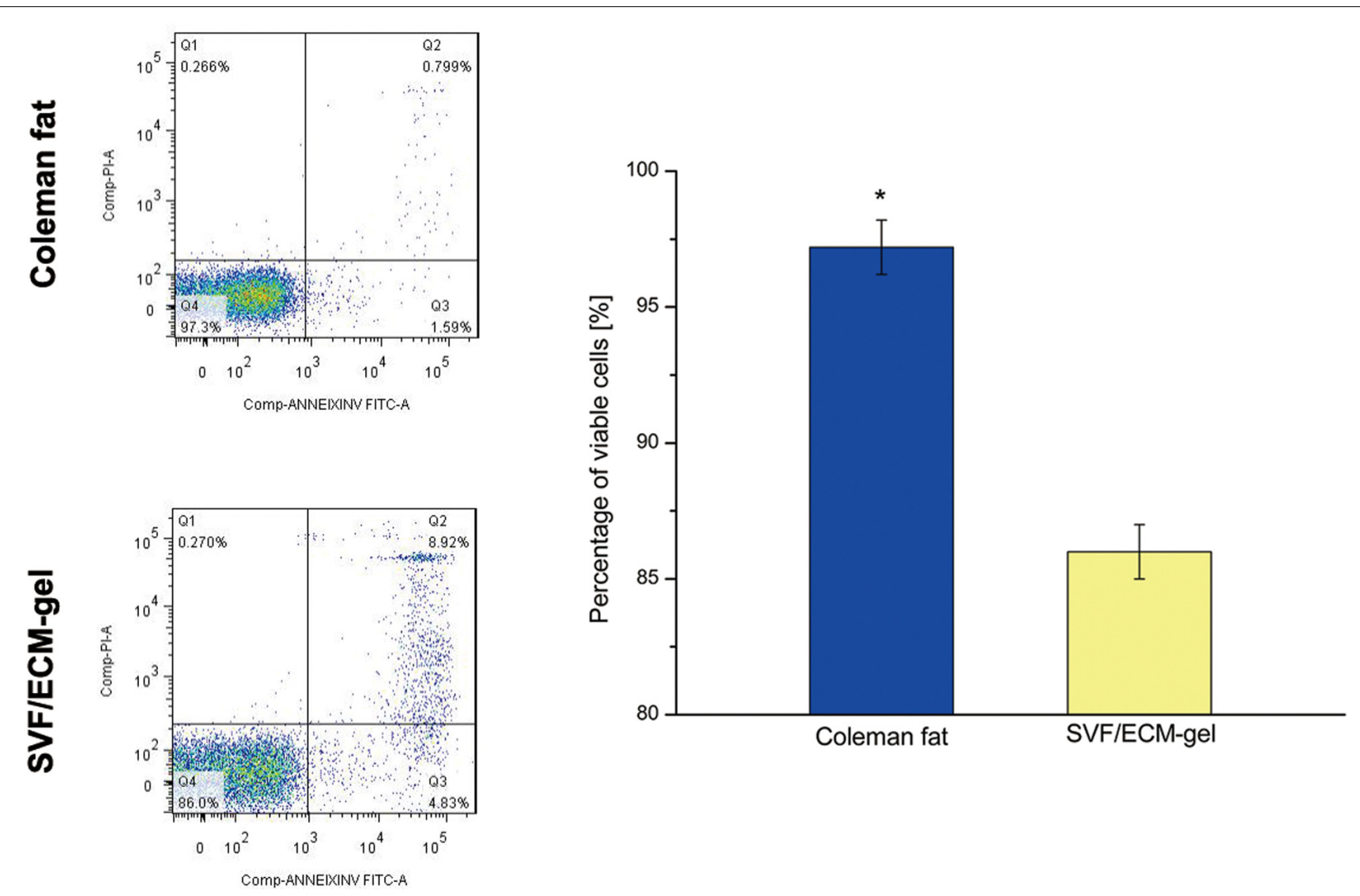

FIGURE 6 | SVF cell activity of the fat grafts. The apoptosis of SVF cells was detected using flow cytometry with Annexin V/PI double staining in Coleman fat and the SVF/ECM gel. * $P<0.05$.

of mature adipocytes, leading to their death. Therefore, the Coleman fat without mechanical isolation had a relatively higher number of total living cells.

Accurately assessing biological changes in cells subject to a mechanical isolation technique is essential. Based on the findings of Zimmerlin et al. (2013) and Banyard et al. (2016), we used a more precise combination of phenotypic markers to identify the proportion of cell subsets among the SVF cells. We used flow cytometry to analyze the expression of each cell surface marker and the change in the proportion of each cell subpopulation in the improved SVF/ECM gel. Although the total number of living cells in the Coleman fat was significantly higher than that in the SVF/ECM gel, the proportions of cells expressing phenotypic (CD34, CD13, CD73, and CD146) and hematopoietic (CD45 and CD31) markers of mesenchymal stem cells were significantly increased in the SVF/ECM gel. This result is consistent with the findings of Banyard et al. (2016). It is likely that the mechanical shear stress liberated many of the SVF cells from the adipose matrix inherent in fat. We found that the relative proportions of ASCs in the unfiltered fat were significantly increased, which is consistent with the results of Yao et al. (2017) and Lombardo and Tamburino (2019). Therefore, the surviving cell population with greater regenerative capacity may account for the favorable results of transplantation in the
SVF/ECM gel grafts. Interestingly, the decrease in the proportion of EPCs contrasts with the results of Yao et al. (2017), which may indicate their inaccurate recognition of endothelial cells based only on the $\mathrm{CD} 45^{-} \mathrm{CD} 34^{+} \mathrm{CD} 31^{+}$phenotype. We added CD146 as a marker to labeled EPCs to better demonstrate the capacity for neovascularization. In addition, the decrease in the relative proportion of EPCs is likely to be influenced by the increase in the relative proportion of other cells such as mesenchymal stem cells and SA-ASCs.

In contrast to other fat preparation methods, enzyme digestion and filtration are not required for the preparation of SVF/ECM gel. Mechanical shear force alone can destroy mature adipocytes, and oil drops are removed during centrifugation, thus reducing the potential inflammatory reaction in the fat grafts and possible postoperative complications. In addition, we found that the relative proportion of cell subpopulations of the SVF mixed cell group in the fat increased under the shear force. These two findings indicate the potential advantages of the clinical transplantation of SVF/ECM gel compared to Coleman fat. The primary limitation of our study is that our results were all based on image observations, without in-depth molecularlevel research. Specifically, the signaling pathways through which mechanical isolation affects the ability of cell proliferation and differentiation along with the relevant key proteins influenced 


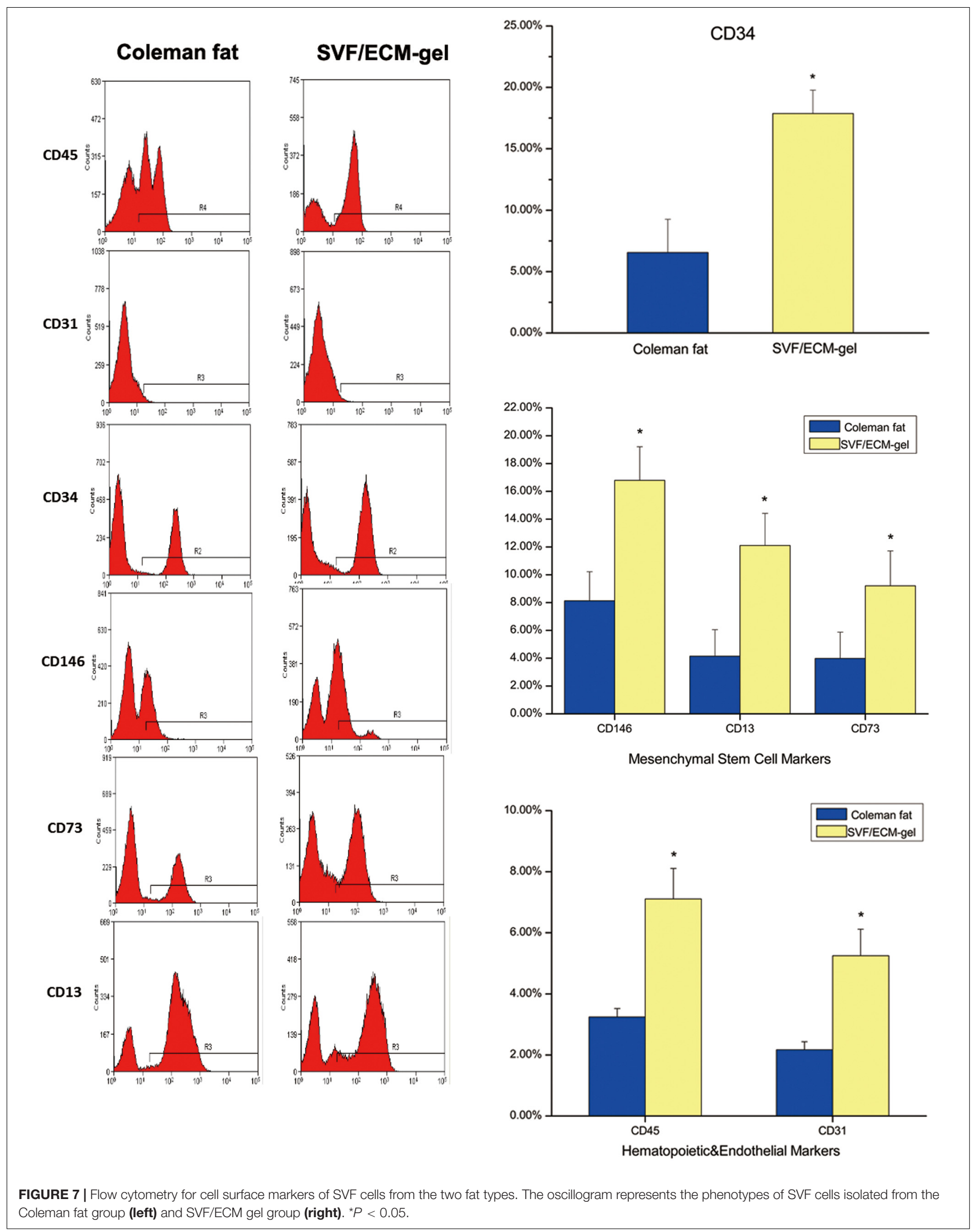




\section{Stromal Vascular Fraction Subpopulations}

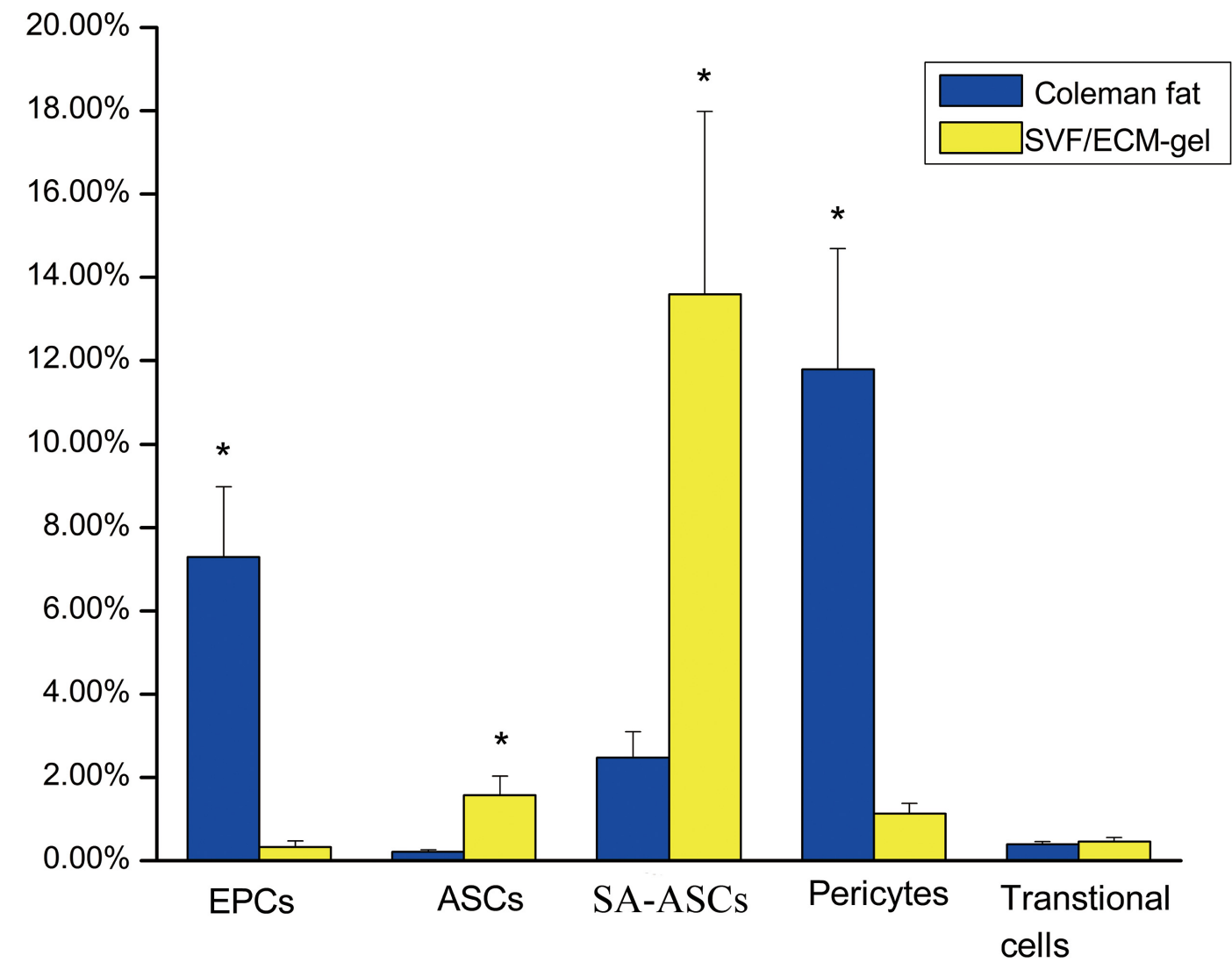

FIGURE 8 | Flow cytometry of the proportions of stem cell subsets in the suspension of SVF cells from the two fat grafts. ASC, adipose-derived stem cell; EPS, endothelial progenitor cell. ${ }^{\star} P<0.05$.

by this process remain unclear. Therefore, the mechanism underlying the observed effects warrants further investigations.

\section{CONCLUSION}

Mechanical isolation is not an inert process and is more complex than the simple decomposition and refinement of fat tissue for transplantation. In particular, the application of shear force could enhance the pluripotency of cells, and this phenotypic advantage may improve fat retention in vivo. Importantly, this study demonstrates the direct biological effects of a physical transformation process with clinical application potential to benefit patients. These effects can help achieve efficient and stable volume filling, while providing an enriched source of regeneration-related cells for cell therapy, thereby advancing the field of clinical tissue repair and reconstruction.

\section{DATA AVAILABILITY STATEMENT}

The original contributions presented in the study are included in the article/supplementary material. Further inquiries can be directed to the corresponding author.

\section{ETHICS STATEMENT}

The studies involving human participants were reviewed and approved by the Ethics Committee of Guangdong Woman and Children Hospital. The patients/participants provided their written informed consent to participate in this study. The animal study was reviewed and approved by the Ethics Committee of Guangdong Woman and Children Hospital.

\section{AUTHOR CONTRIBUTIONS}

YY independently performed the work under the guidance of JJ. JZ and MT provided technical support during tissue slice observation. KH performed the statistical analysis. All authors contributed to the article and approved the submitted version.

\section{ACKNOWLEDGMENTS}

We would like to thank Editage (www.editage.cn) for English language editing. 


\section{REFERENCES}

Banyard, D. A., Sarantopoulos, C. N., Borovikova, A. A., Qiu, X., Wirth, G. A., Paydar, K. Z., et al. (2016). Phenotypic analysis of stromal vascular fraction after mechanical shear reveals stress-induced progenitor populations. Plast. Reconstr. Surg. 138, 237e-247e. doi: 10.1097/PRS.0000000000002356

Bashir, M. M., Sohail, M., Ahmad, F. J., and Choudhery, M. S. (2019). Preenrichment with adipose tissue-derived stem cells improves fat graft retention in patients with contour deformities of the face. Stem Cells Int. 2019, 5146594. doi: 10.1155/2019/5146594

Billings, E., and May, J. (1989). Historical review and present status of free fat graft autotransplantation in plastic and reconstructive surgery. Plast. Reconstr. Surg. 83, 368-381. doi: 10.1097/00006534-198902000-00033

Cai, W., Yu, L. D., Tang, X., and Shen, G. (2018). The stromal vascular fraction improves maintenance of the fat graft volume: a systematic review. Ann. Plast. Surg. 81, 367-371. doi: 10.1097/SAP.0000000000001589

Canizares, O. Jr., Thomson, J. E., Allen, R. J. Jr., Davidson, E. H., Tutela, J. P., Saadeh, P. B., et al. (2017). The effect of processing technique on fat graft survival. Plast. Reconstr. Surg. 140, 933-943. doi: 10.1097/PRS. 0000000000003812

Cansancao, A. L., Conde-Green, A., David, J. A., and Vidigal, R. A. (2019). Subcutaneous-only gluteal fat grafting: a prospective study of the long-term results with ultrasound analysis. Plast. Reconstr. Surg. 143, 447-451. doi: 10. 1097/PRS.0000000000005203

Chen, L., Wang, Z. C., Ma, J. J., Sun, W. J., Wang, S. W., Gu, Z. C., et al. (2019). Autologous nanofat transplantation accelerates foot wound healing in diabetic rats. Regen. Med. 14, 231-241. doi: 10.2217/rme-2018-0169

Chen, X., Deng, Z., He, Y., Lu, F., and Yuan, Y. (2020). Mechanical strain promotes proliferation of ADSCs through the integrin betal-mediated RhoA/MLC pathway. Tissue Eng. Part A 26, 939-952. doi: 10.1089/ten.TEA.2019.0266

Cohen, S. R., Hewett, S., Ross, L., Delaunay, F., Goodacre, A., Ramos, C., et al. (2017). Regenerative cells for facial surgery: biofilling and biocontouring. Aesthet. Surg. J. 37, S16-S32. doi: 10.1093/asj/sjx078

Coleman, S. (2006). Structural fat grafting: more than a permanent filler. Plast. Reconstr. Surg. 118, 108S-120S. doi: 10.1097/01.prs.0000234610.81672.e7

Cucchiani, R., and Corrales, L. (2016). The effects of fat harvesting and preparation, air exposure, obesity, and stem cell enrichment on adipocyte viability prior to graft transplantation. Aesthet. Surg. J. 36, 1164-1173. doi: 10.1093/asj/sjw106

Doi, K., Ogata, F., Eto, H., Kato, H., Kuno, S., Kinoshita, K., et al. (2015). Differential contributions of graft- and host-derived cells in tissue regeneration/remodeling after fat grafting. Plast. Reconstr. Surg. 135, 16071617. doi: 10.1097/PRS.0000000000001292

Dong, Z., Peng, Z., Chang, Q., Zhan, W., Zeng, Z., Zhang, S., et al. (2015). The angiogenic and adipogenic modes of adipose tissue after free fat grafting. Plast. Reconstr. Surg. 135, 556e-567e. doi: 10.1097/PRS.0000000000000965

Eto, H., Kato, H., Suga, H., Aoi, N., Doi, K., Kuno, S., et al. (2012). The fate of adipocytes after nonvascularized fat grafting: evidence of early death and replacement of adipocytes. Plast. Reconstr. Surg. 129, 1081-1092. doi: 10.1097/ PRS.0b013e31824a2b19

Fraser, J. K., Hedrick, M. H., and Cohen, S. R. (2011). Oncologic risks of autologous fat grafting to the breast. Aesthet. Surg. J. 31, 68-75. doi: 10.1177/ 1090820X10390922

Gebremeskel, S., Gencarelli, J., Gareau, A. J., Levatte, T., Dugandzic, A., Johnston, B., et al. (2019). Promotion of primary murine breast cancer growth and metastasis by adipose-derived stem cells is reduced in the presence of autologous fat graft. Plast. Reconstr. Surg. 143, 137-147. doi: 10.1097/PRS. 0000000000005142

Gontijo-de-Amorim, N. F., Charles-De-Sa, L., and Rigotti, G. (2017). Mechanical supplementation with the stromal vascular fraction yields improved volume retention in facial lipotransfer: a 1-year comparative study. Aesthet. Surg. J. 37, 975-985. doi: 10.1093/asj/sjx115

Guo, J., Nguyen, A., Banyard, D. A., Fadavi, D., Toranto, J. D., Wirth, G. A., et al. (2016). Stromal vascular fraction: a regenerative reality? Part 2: mechanisms of regenerative action. J. Plast. Reconstr. Aesthet. Surg. 69, 180-188. doi: 10.1016/j. bjps.2015.10.014

Hong, K. Y., Kim, I. K., Park, S. O., Jin, U. S., and Chang, H. (2019). Systemic administration of adipose-derived stromal cells concurrent with fat grafting. Plast. Reconstr. Surg. 143, 973e-982e. doi: 10.1097/PRS.0000000000005513
Kato, H., Mineda, K., Eto, H., Doi, K., Kuno, S., Kinoshita, K., et al. (2014). Degeneration, regeneration, and cicatrization after fat grafting: dynamic total tissue remodeling during the first 3 months. Plast. Reconstr. Surg. 133, 303e313e. doi: 10.1097/PRS.0000000000000066

Kim, K., Fan, Y., Lin, G., Park, Y. K., Pak, C. S., Jeong, J. H., et al. (2019). Synergistic effect of adipose-derived stem cells and fat graft on wrinkles in aged mice. Plast. Reconstr. Surg. 143, 1637-1646. doi: 10.1097/PRS.0000000000005625

Krumboeck, A., Giovanoli, P., and Plock, J. A. (2013). Fat grafting and stem cell enhanced fat grafting to the breast under oncological aspectsrecommendations for patient selection. Breast 22, 579-584. doi: 10.1016/j. breast.2013.05.006

Li, K., Li, F., Li, J., Wang, H., Zheng, X., Long, J., et al. (2017). Increased survival of human free fat grafts with varying densities of human adipose-derived stem cells and platelet-rich plasma. J. Tissue Eng. Regen. Med. 11, 209-219. doi: 10.1002/term.1903

Li, X., Kubiak, C. A., Yang, X., Kemp, S. W. P., Cederna, P. S., and Ma, J. (2019). Forehead fat grafting: asian facial contouring and augmentation. Plast. Reconstr. Surg. 144, 1057-1065. doi: 10.1097/PRS.0000000000006122

Li, Y., Wu, M., Zhang, Z., Xia, J., Wang, Z., Chen, X., et al. (2019). Application of external force regulates the migration and differentiation of adipose-derived stem/progenitor cells by altering tissue stiffness. Tissue Eng. Part A 25, 16141622. doi: 10.1089/ten.TEA.2019.0046

Lombardo, G. A. G., and Tamburino, S. (2019). The unfiltered nanofat: a great source of staminal cells. Ann. Plast. Surg. 83, 488. doi: 10.1097/SAP. 0000000000001958

Nava, M. M., Raimondi, M. T., and Pietrabissa, R. (2012). Controlling self-renewal and differentiation of stem cells via mechanical cues. J. Biomed. Biotechnol. 2012:797410. doi: 10.1155/2012/797410

Oranges, C. M., Striebel, J., Tremp, M., Madduri, S., Kalbermatten, D. F., Harder, Y., et al. (2019). The preparation of the recipient site in fat grafting: a comprehensive review of the preclinical evidence. Plast. Reconstr. Surg. 143, 1099-1107. doi: 10.1097/PRS.0000000000005403

Orbay, H., Hinchcliff, K. M., Charvet, H. J., and Sahar, D. E. (2018). fat graft safety after oncologic surgery: addressing the contradiction between in vitro and clinical studies. Plast. Reconstr. Surg. 142, 1489-1499. doi: 10.1097/PRS. 0000000000004992

Pallua, N., and Kim, B. S. (2020). Microfat and lipoconcentrate for the treatment of facial scars. Clin. Plast. Surg. 47, 139-145. doi: 10.1016/j.2019.08.010

Qiu, L., Su, Y., Zhang, D., Song, Y., Liu, B., Yu, Z., et al. (2016). Identification of the centrifuged lipoaspirate fractions suitable for postgrafting survival. Plast. Reconstr. Surg. 137, 67e-76e. doi: 10.1097/PRS.0000000000001883

Rohrich, R. J., Sanniec, K., and Afrooz, P. N. (2018). Autologous fat grafting to the chin: a useful adjunct in complete aesthetic facial rejuvenation. Plast. Reconstr. Surg. 142, 921-925. doi: 10.1097/PRS.0000000000004817

Sese, B., Sanmartin, J. M., Ortega, B., Matas-Palau, A., and Llull, R. (2019). Nanofat cell aggregates: a nearly constitutive stromal cell inoculum for regenerative site-specific therapies. Plast. Reconstr. Surg. 144, 1079-1088. doi: 10.1097/PRS. 0000000000006155

Streit, L., Jaros, J., Sedlakova, V., Sedlackova, M., Drazan, L., Svoboda, M., et al. (2017). A comprehensive in vitro comparison of preparation techniques for fat grafting. Plast. Reconstr. Surg. 139, 670e-682e. doi: 10.1097/PRS. 0000000000003124

Sun, Y., Chen, C. S., and Fu, J. (2012). Forcing stem cells to behave: a biophysical perspective of the cellular microenvironment. Annu. Rev. Biophys. 41, 519-542. doi: 10.1146/annurev-biophys-042910-155306

Tang, H. H., Han, X. L., Debels, H., Tan, B., Palmer, J., Poon, C., et al. (2018). Fate of free fat grafts with or without adipogenic adjuncts to enhance graft outcomes. Plast. Reconstr. Surg. 142, 939-950. doi: 10.1097/PRS.0000000000004739

Tonnard, P., Verpaele, A., and Carvas, M. (2020). Fat grafting for facial rejuvenation with nanofat grafts. Clin. Plast. Surg. 47, 53-62. doi: 10.1016/j.cps. 2019.08.006

Tonnard, P., Verpaele, A., Peeters, G., Hamdi, M., Cornelissen, M., and Declercq, H. (2013). Nanofat grafting: basic research and clinical applications. Plast. Reconstr. Surg. 132, 1017-1026. doi: 10.1097/PRS.0b013e31829fe1b0

Trivisonno, A., Alexander, R. W., Baldari, S., Cohen, S. R., Di Rocco, G., Gentile, P., et al. (2019). Intraoperative strategies for minimal manipulation of autologous adipose tissue for cell- and tissue-based therapies: concise review. Stem Cells Transl. Med. 8, 1265-1271. doi: 10.1002/sctm.19-0166 
Tuin, A. J., Domerchie, P. N., Schepers, R. H., Willemsen, J. C., Dijkstra, P. U., Spijkervet, F. K., et al. (2016). What is the current optimal fat grafting processing technique? A systematic review. J. Craniomaxillofac. Surg. 44, 45-55. doi: 10. 1016/j.jcms.2015.10.021

Uyulmaz, S., Sanchez Macedo, N., Rezaeian, F., Giovanoli, P., and Lindenblatt, N. (2018). Nanofat grafting for scar treatment and skin quality improvement. Aesthet. Surg. J. 38, 421-428. doi: 10.1093/asj/sjx183

van Dongen, J. A., Tuin, A. J., Spiekman, M., Jansma, J., Van Der Lei, B., and Harmsen, M. C. (2018). Comparison of intraoperative procedures for isolation of clinical grade stromal vascular fraction for regenerative purposes: a systematic review. J. Tissue Eng. Regen. Med. 12, e261-e274. doi: 10.1002/term. 2407

Verpaele, A., Tonnard, P., Jeganathan, C., and Ramaut, L. (2019). Nanofat needling: a novel method for uniform delivery of adipose-derived stromal vascular fraction into the skin. Plast. Reconstr. Surg. 143, 1062-1065. doi: 10.1097/PRS. 0000000000005455

Wei, S., Liu, W., Gundogan, B., Moscoso, A. V., Orgill, D. P., and Giatsidis, G. (2019). Delayed postconditioning with external volume expansion improves survival of adipose tissue grafts in a murine model. Plast. Reconstr. Surg. 143, 99e-110e. doi: 10.1097/PRS.0000000000005167

Yao, Y., Dong, Z., Liao, Y., Zhang, P., Ma, J., Gao, J., et al. (2017). Adipose extracellular matrix/stromal vascular fraction gel: a novel adipose tissue-derived injectable for stem cell therapy. Plast. Reconstr. Surg. 139, 867-879. doi: 10.1097/ PRS.0000000000003214

Ye, Y., Liao, Y., Lu, F., and Gao, J. (2017). Daily suction provided by external volume expansion inducing regeneration of grafted fat in a murine model. Plast. Reconstr. Surg. 139, 392e-402e. doi: 10.1097/PRS.0000000000003012
Yuan, Y., Zhang, S., Gao, J., and Lu, F. (2015). Spatial structural integrity is important for adipose regeneration after transplantation. Arch. Dermatol. Res. 307, 693-704. doi: 10.1007/s00403-015-1574-y

Zhang, J., Bai, X., Zhao, B., Wang, Y., Su, L., Chang, P., et al. (2016). Allogeneic adipose-derived stem cells promote survival of fat grafts in immunocompetent diabetic rats. Cell Tissue Res. 364, 357-367. doi: 10.1007/s00441-015-2334-1

Zhang, Z., Cai, J., Li, Y., He, Y., Dong, Z., Dai, J., et al. (2020). External volume expansion adjusted adipose stem cell by shifting the ratio of fibronectin to laminin. Tissue Eng. Part A 26, 66-77. doi: 10.1089/ten.TEA.2019. 0095

Zhou, Y., Wang, J., Li, H., Liang, X., Bae, J., Huang, X., et al. (2016). Efficacy and safety of cell-assisted lipotransfer: a systematic review and meta-analysis. Plast. Reconstr. Surg. 137, 44e-57e. doi: 10.1097/PRS.0000000000001981

Zimmerlin, L., Donnenberg, V. S., Rubin, J. P., and Donnenberg, A. D. (2013). Mesenchymal markers on human adipose stem/progenitor cells. Cytometry A 83, 134-140. doi: 10.1002/cyto.a.22227

Conflict of Interest: The authors declare that the research was conducted in the absence of any commercial or financial relationships that could be construed as a potential conflict of interest.

Copyright (c) $2021 \mathrm{Ye}, \mathrm{Zou}, \mathrm{Tan}, \mathrm{Hu}$ and Jiang. This is an open-access article distributed under the terms of the Creative Commons Attribution License (CC BY). The use, distribution or reproduction in other forums is permitted, provided the original author(s) and the copyright owner(s) are credited and that the original publication in this journal is cited, in accordance with accepted academic practice. No use, distribution or reproduction is permitted which does not comply with these terms. 